\title{
The LEP2 Superconducting RF System
}

\author{
J. TUCKMANTEL (for the LEP sc. cavity team) \\ CERN, Geneva, Switzerland
}

\begin{abstract}
The LEP2 superconducting RF system supplies at present about $2600 \mathrm{MV}$ per turn to the electrons and positrons of the LEP beams, giving a beam energy largely above $90 \mathrm{GeV}$ to create the desired massive $\mathrm{W}^{ \pm}$-pairs for physics. It is by far the largest such system world wide and a further upgrade is still under way to increase the $\mathrm{W}^{ \pm}$production rate and cover new physics grounds. More than $10 \mathrm{MW}$ RF power were routinely transmitted to the beam during last year's smooth operation. We will present the technical parts of this system: the cavities, couplers, tuners, cryostats, the cryogenics and the RF generation and distribution. We will also report on operational experience and future plans.
\end{abstract}

\section{INTRODUCTION}

The LEP machine (Large Electron Positron collider) [1] was built at CERN to produce $\mathrm{Z}^{\circ}$ bosons and in an upgraded stage $\mathrm{W}^{ \pm}$boson-pairs. Collisions of counter rotating bunched electron and positron beams create the desired bosons at a few dedicated points. Around those four - spaced by a quarter machine circumference - large particle detectors have been constructed. The circulating electrons and positrons are deflected in the bending magnets and emit a broad spectrum of synchrotron radiation quanta in the one-MeV range. The corresponding energy loss has to be replaced by an accelerating radio frequency (RF) system. The losses per turn increase as the 4th power of the beam energy and decrease linearly with the radius of the machine, imposing the large circumference of about $27 \mathrm{~km}$ for LEP, at present the largest machine in the world. The same tunnel will house in the future the Large Hadron Collider [2].

$\mathrm{Z}^{\mathrm{o}}$-bosons were created at a beam energy of $45 \mathrm{GeV}$. The necessary accelerating voltage of about $280 \mathrm{MV}$ was supplied by 128 copper cavities operating at $352 \mathrm{MHz}$. To produce $\mathrm{W}^{ \pm}$boson-pairs in reasonable quantities the beam energy has to be increased to at least about $84 \mathrm{GeV}$, the $\mathrm{W}^{ \pm}$-pair production rate increasing with beam energy above the $80.5 \mathrm{GeV}$ threshold energy.

The corresponding accelerating voltage in LEP had thus to be boosted by more than an order of magnitude (the LEP2 program). A superconducting (sc) RF system can supply such a voltage with reasonable electricity cost. Since the same voltage can be delivered by fewer sc cavities as compared to copper cavities and since the beam hole openings of sc cavities are larger, the sc system presents also a lower impedance. This is an essential feature to achieve a high circulating beam current and a high luminosity.

Manuscript received September 14, 1998

\section{MODULE COMPONENTS}

The LEP2 RF system uses so-called modules composed of four 4-cell cavities and their ancillaries housed in a common cryostat and having a common He supply. Each such module is designed to supply $40 \mathrm{MV}$ to the beam, the cavities being excited at $6 \mathrm{MV} / \mathrm{m}$.

\section{A. The Cavities}

CERN started a small R\&D program in 1979. The first milestone was a bulk $\mathrm{Nb} 5$-cell $500 \mathrm{MHz}$ cavity tested successfully in the PETRA machine (DESY, Hamburg) in 1983 [3]. Based on the gained experience a 4-cell cavity with all couplers on the beam tubes was designed [4]. The chosen frequency was $352.2 \mathrm{MHz}$ like the existing copper system for compatibility reasons. The first bare $\mathrm{Nb}$ 4-cell prototype cavity was realized in 1986 .

A thermal conductivity higher than 'reactor grade' $\mathrm{Nb}$ increases the 'defect tolerance' of the cavities. Test cavities made at CERN from specially refined (high RRR) $\mathrm{Nb}$ led to significantly higher gradients. In 1991 - after the smooth commissioning of LEP - a first 4-cavity module using prototype cavities of such material was installed and operated successfully in LEP. Five similar modules were ordered from industry and four of them are still working in LEP.

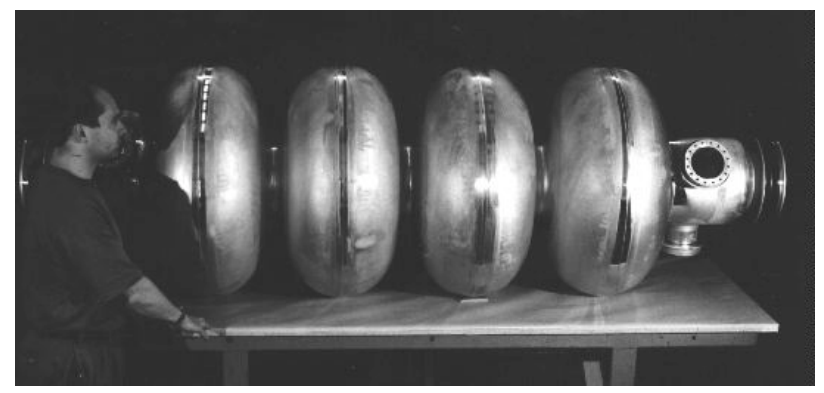

Fig. 1: 4-cell Copper substrate cavity (before sputtering). 256 of those are at present installed in LEP.

At low temperatures OFHC copper is a much better thermal conductor than even high RRR $\mathrm{Nb}$. Thus CERN has developed the technology to deposit (by sputtering) a $\mathrm{Nb}$ film on ready shaped copper (substrate) cavities ( $\mathrm{Nb} / \mathrm{Cu}$ cavities). The film is about $1 \mu \mathrm{m}$ thick (20 penetration depths) but 'thermally invisible'; its weight is only about $50 \mathrm{~g}$. This layer has to be produced in one single step and free of defects for the whole surface of about $6 \mathrm{~m}^{2}$ (there is no technique for local defect recovery). No $\mathrm{Nb} / \mathrm{Cu}$ cavity was found to be limited by a hard quench. Compared to bulk $\mathrm{Nb}$ cavities $\mathrm{Nb} / \mathrm{Cu}$ cavities show higher Q-values at low field but a 
steeper decrease of $\mathrm{Q}$ with rising field (for an operation at $4.5 \mathrm{~K}$ ). The operational field of $6 \mathrm{MV} / \mathrm{m}$ is in the cross-over range. Another advantage is that $\mathrm{Nb} / \mathrm{Cu}$ cavities are insensitive to the earth magnetic field - a complicated magnetic shielding being expendable - and they are easy to condition and operate due to the absence of hard quenches. This technology has been transferred meanwhile to three European companies; each of them supplied about $1 / 3$ of the modules operating in LEP now.

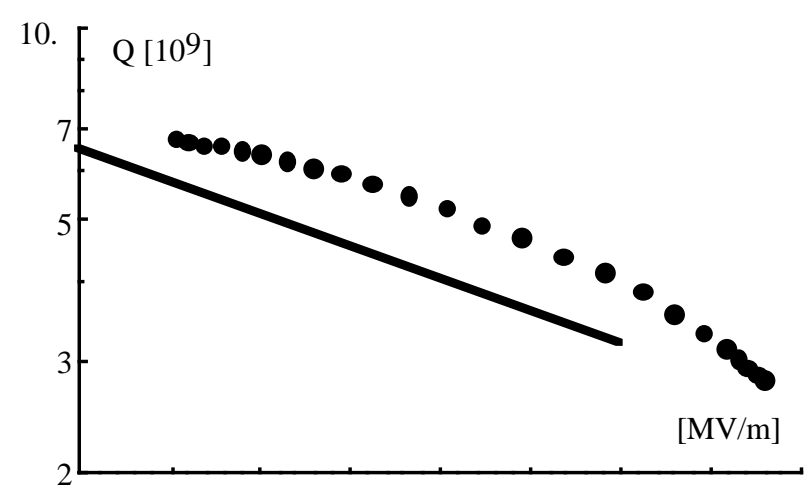

Fig 2. Typical $\mathrm{Q}(\mathrm{E})$ with specifications (thick line) for a $\mathrm{Nb} / \mathrm{Cu}$ cavity

\section{B. The Cryostat}

The cryostat [5] (see fig. 3) is composed of independent cylinder shaped units linked by large Helicoflex® vacuum joints. Each unit houses a 4-cell cavity with its ancillaries. Any number of units can be joined to form a module with common insulation vacuum and common He supply. Cavities are connected by bellows as are the common helium gas and liquid collectors. In LEP we use exclusively 4-cavity modules (see fig 6,7), however, in the SPS (the LEP injector) two 2cavity modules are operating.

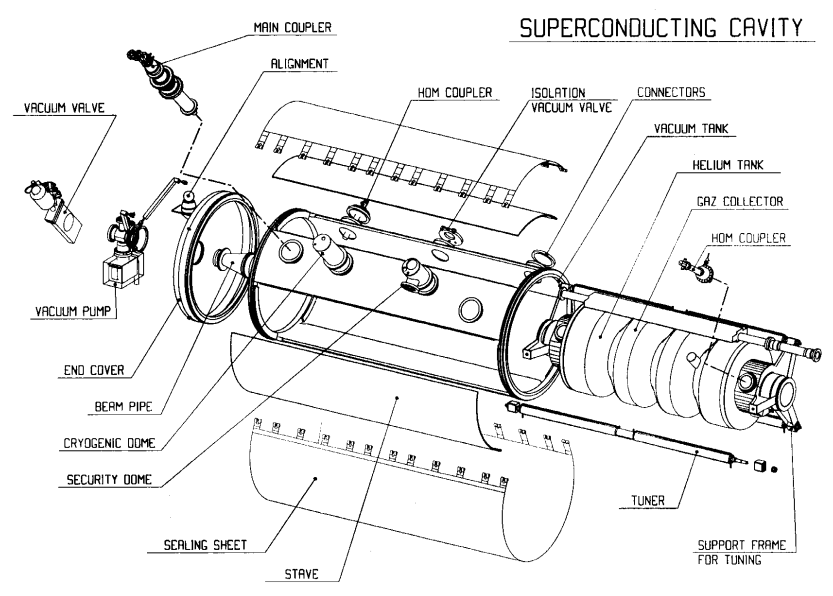

Fig. 3: Exploded view of one unit: He-tank (right) with the cavity inside, tuners (around), HOM- and power couplers (left). Steel sheets (top and bottom) close the insulation vacuum
The vacuum vessel of each unit consists of two cylindrical stiff rings at each end connected by barrel staves. Two of these are welded to the rings and carry all ports for helium and electrical connections, the other ones are (easily) dismountable. Vacuum tightness is assured by a pair of stainless steel sheets pulled around and pressed against two Orings running along the openings in the frame. This technique allows opening and easy access to components for repair or exchange, even on cavities already installed in the accelerator.

\section{The Tuners}

Tuning is realized by longitudinal elastic cavity deformation. The cavities (with their welded He-tank) carry at each end three arms spaced by $120^{\circ}$ and protruding outside the He-tank diameter. Each pair of corresponding arms on both sides is connected by a 'tuner bar' [6] (see fig. 3) defining the cavity length. The coarse cavity frequency is set by spacers. The tuner bar itself consists of a tube of $60 \mathrm{~mm}$ diameter made of heat treated nickel. It is surrounded by a single layer He gas cooled coil. In changing the current (0-5 A), the length of the Ni-bar can be varied precisely by magnetostriction (MS) up to a stroke of $50 \mu \mathrm{m}$ corresponding to $2 \mathrm{kHz}$ detuning. This MS tuner has a rise time of about $50 \mathrm{~ms}$.

This MS system is precise and fast but does not have a large stroke. Therefore thermal expansion is used additionally. Cold evaporated He-gas is injected on both sides of the Nitube and channeled towards a common central outlet. In the center of the Ni-tube a heater is used to heat and thus expand the Ni-tube. The resulting slow frequency change (a few Hz/s) amounts to more than $50 \mathrm{kHz}$ for a tuner bar center temperature limited to $300 \mathrm{~K}$.

The RF system detects deviations from the nominal frequency and acts immediately on the fast MS tuner. The averaged MS tuner position is kept in the middle of the range using the slower thermal tuner. If the RF is off, the thermal tuner control is taken over by temperature regulation using an individually matched set value, keeping the frequency about constant.

This tuning system is based on a device without mechanical movements - thus it cannot get stuck - and it does not need endplates as mechanical strong point. This tuner was found to work without real problems, the only drawback being the limited speed of the thermal tuner.

\section{The Higher Order Mode Coupling System}

The bunches in LEP excite higher order modes (HOM) in all cavities and by resonant build-up the beam may get lost, thus sufficient damping of the modes has to be guaranteed. Therefore both beam tubes carry a HOM coupler. An azimuthal angle of $115^{\circ}$ between the two couplers ensures safe coupling also to multipole modes. The extracted energy has to 
be conducted through the insulation vacuum to be transformed into heat outside the cryostat.

HOM couplers are designed to couple strongly to all modes, so a stop filter has to be incorporated to prevent power extraction from the accelerating mode. Resonant filters carry high (reactive) currents. Therefore the main part of the HOM coupler (hook and cap) has to be superconducting itself and is made of bulk $\mathrm{Nb}$ sheet.

The HOM couplers at CERN were designed dismountable, fitting into ports on the cavity beam tubes. The coupler is of the hook type [7], the hook forming a series resonator tuned to $352 \mathrm{MHz}$ which short-circuits the picked up accelerating mode component to ground. For cooling, liquid $\mathrm{He}$ is injected through the lateral support and gaseous He can escape by the same channel. This lateral support represents a short circuit for DC but is situated such that it forms part of a second filter enhancing the coupling in a frequency region including a few important modes.

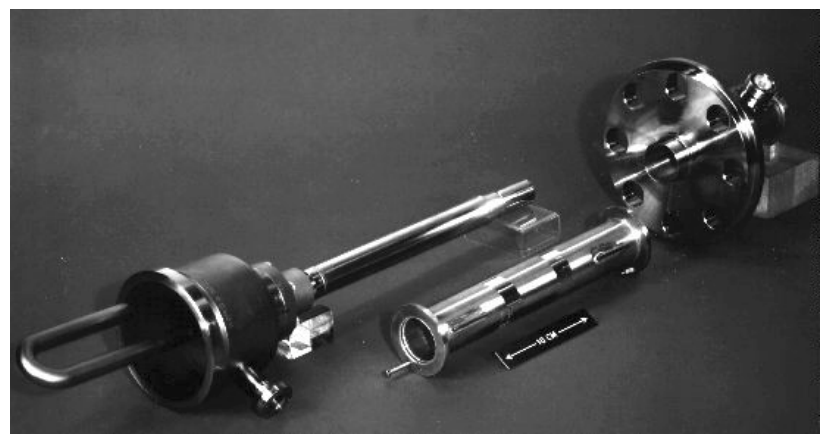

Fig. 4: Hook-type HOM coupler made of $\mathrm{Nb}$ (left) and parts of the rigid coaxial output line

The HOM power is coupled out capacitively and passes a ceramic RF window. This window separates the cavity vacuum and the insulation vacuum, thus a rupture of the window under operational vacuum conditions is (nearly) without consequences.

The HOM power transport through the insulation vacuum is made by a rigid coaxial line capable of carrying at least $1 \mathrm{~kW}$ and giving enough margin for the estimated worst case of $600 \mathrm{~W}$ coupled HOM power.

\section{E. The Power Coupler}

The initial design of the power coupler - adapted from the copper cavity system - was apparently largely overdimensioned. However, in the now cold environment multipacting (MP) on the outer conductor of the coaxial line showed up. It could be conditioned away in applying RF, however, after a short interruption or a change of RF power level or phase, MP came back again, an effect for which the name 'deconditioning' was coined.

Tests on a model with local liquid nitrogen cooling gave the right hint [8]: adsorbed gasses (water was a particularly bad representative) on the surface were released during conditioning but instead of being pumped away as for a warm line were soon captured again on the cold surface in the vicinity. Therefore several construction and production measures were taken [9] to reduce RF heating of the ceramic window - which has a spongiform structure and releases gasses when heated - and the windows were baked to about $200^{\circ} \mathrm{C}$ once the couplers were installed on the cavity before cooling down.

These measures eased conditioning considerably but only the application of a DC bias voltage of about $-2.5 \mathrm{kV}$ on the center conductor - modifying the kinematics of the electron tracks - finally removed multipacting definitely. To separate DC and RF, a special capacitor - produced at CERN - had to be included into the construction of the doorknob transition.

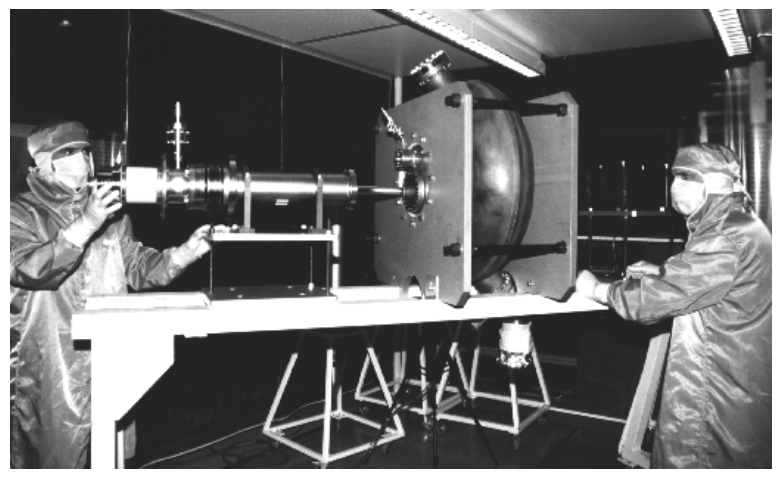

Fig. 5: Cleanroom preparation of a power coupler (pair) for high power processing

However, application of DC voltage without preconditioning may cause breakthrough. Therefore all couplers were conditioned after fabrication as pairs on a (warm) test stand and almost all modules were operated under 'tunnel conditions' on a (cold) test stand before installation into LEP.

Once these production and preparation procedures applied, no more power coupler problems were encountered during operation.

\section{F. Series Production and Acceptance Tests}

Cavity production [10] started from $900 \times 900 \mathrm{~mm}$ and $3.5 \mathrm{~mm}$ thick OHFC copper sheets which were first inspected and checked for iron inclusions. Half cells were produced by spinning and the damage layer removed by electropolishing $(120 \mu \mathrm{m})$. Two half cells were then welded together by electron beam welding using an internal gun and the resulting cell was tested for its RF frequency. Four cells and the prefabricated cutoff tubes with ports were afterwards welded together with the same technique. This copper cavity was adjusted by inelastic longitudinal deformation to $5 \%$ tolerance in the field profile and the resonant frequency to $50 \mathrm{kHz}$. The cavity underwent a weak chemical polishing, after which the $\mathrm{Nb}$ clad cathode of the magnetron was inserted into the cavity in a clean room and the $1 \mu \mathrm{m}$ film sputtered; the cavity was 
then rinsed under a laminar air flow unit with ultra pure water and finally dried.

Since this $\mathrm{Nb}$ film is the most critical element, cavities were pre-tested at CERN at $\mathrm{LHe}$ temperature. If a cavity showed a failure in the $\mathrm{Nb}$ layer, it was stripped off at CERN. Cavities were in any case sent back; if they had been accepted, the He-tank was welded around, otherwise a stripped cavity was deposited again. New coatings could be repeated 3-4 times. It was found that the primary success rate increased during the project reaching about $75 \%$ at the end. At the factory units including also vacuum vessels and tuners were assembled, combined into modules and closed with curved end lids. Connecting the cavities together was done in a class 100 clean room to avoid dust particles entering the cavities' volumes. The GHe collectors of the four units as well as the inlet $\mathrm{LHe}$ collector were connected such that there exists only one common inlet and one common outlet for helium. These modules - without power and HOM couplers - were shipped to CERN where the contractual $\mathrm{Q}(\mathrm{E})$ curve was measured.

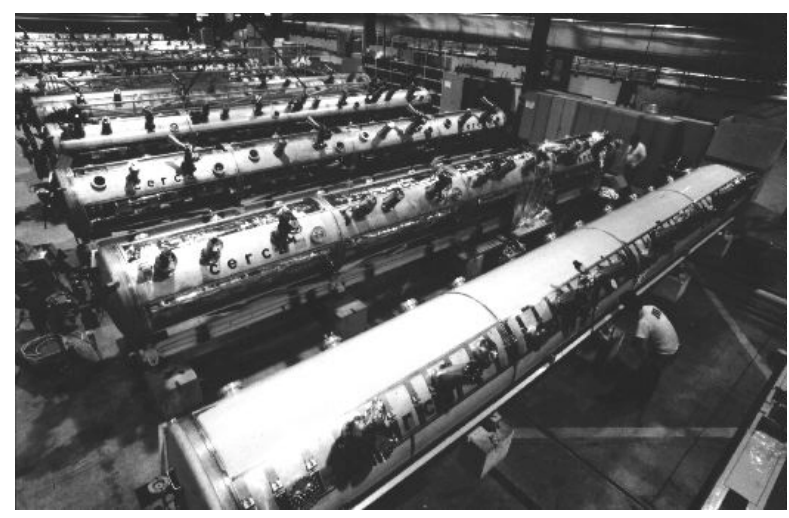

Fig. 6: 'Factory' at CERN to upgrade accepted modules with HOM- and power couplers and cryogenic supply lines

Rejection rate of these modules was below $10 \%$, and all those modules could be made working with rather modest repairs, either water rinsing or re-deposition of a single cavity, which asked evidently for (partial) disassembly of the module.

Once accepted, the modules were opened - here the easy access was very valuable - and equipped with HOM and power couplers in the clean room at CERN. A second test with a high power klystron was done, essentially to test and condition the - preprocessed - power couplers. Two modules had to be rejected at this stage probably due to accidents which took place when mounting couplers in the clean room. Both could be recovered with the methods mentioned above. From here modules went into storage, waiting for the next time slot for installation. In LEP, modules were checked out again and conditioned without beam before the start of the physics operation period.

\section{OPERATION IN THE LEP TUNNEL}

\section{A. The Helium Supply System}

The sc modules are grouped in sections - short compared to the machine circumference - left and right of the four interaction points (IP). All modules at one IP are supplied by one cryogenic plant producing about $12 \mathrm{~kW}$ equivalent cooling power at $4.5 \mathrm{~K}$, about $1.5 \mathrm{~kW}$ of it being used to keep all transfer lines cold. As a preparation for the planned maximum energy run in the year 2000 the plants are being upgraded to about $15 \mathrm{~kW}$. The (screw) compressors are on ground level. A coldbox handling the corresponding gas flows is too huge to be housed underground, but a coldbox on ground would be linked by a towering column of LHe to the modules, producing a 'heliostatic' pressure difference of about 1 bar. Therefore a split coldbox is used. The larger part on ground handles the more extended gas flows and produces pressurized gas of about $20 \mathrm{~K}$. This much denser gas is sent down in a cryogenic concentric tube assembly - which handles also the return gas - to the smaller lower coldbox underground where the desired LHe is produced. From this coldbox two main transfer lines branch right and left into the corresponding tunnel sections, the lines being tapped with a flexible line piece at each module.

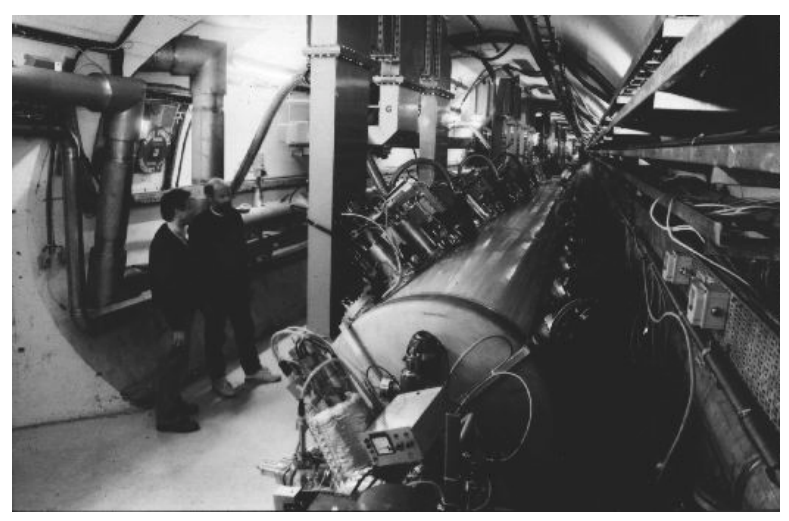

Fig. 7: Module installed in the LEP tunnel. Left of it the RF waveguides, in front a compensated sector valve. The cryogenic transfer line is passing on the left side of the tunnel.

Each module is controlled individually in $\mathrm{He}$ level and pressure by operating the inlet and outlet valves. A bath heater is used to compensate load modifications when changing the RF field, the plants thus having an about constant load.

A cryogenic instability was detected on several operational modules. Investigations revealed that the connecting pieces at the end of the flexible transfer line and the corresponding inlet tube on the module left a small circular gap which could be excited like an organ pipe by the flowing He gas, resonating around $100 \mathrm{~Hz}$. Later all these gaps were filled by a Kapton ${ }^{\circledR}$ foil before introducing the connecting pipes and this type of oscillations disappeared. 


\section{B. The High Power RF System}

The RF power is generated in CW klystrons of 1 MW maximum power (new ones reach $1.3 \mathrm{MW}$ ). Each one is equipped with circulator and load. Power from each klystron is split in 3 levels by 'magic Ts' to feed 8 cavities (two modules).

This choice has the drawback that one generator supplies eight users which makes feedback difficult or sometimes impossible and entails $80 \mathrm{MV}$ loss in case of a klystron trip (plus the induced inverse voltage). On the other hand, a single large klystron is much more efficient and less expensive than a multitude of smaller ones (a powerful argument considering the size of the total system). Also, a very similar system was already in operation for the copper cavities and could thus be adapted with only minor modifications.

To stabilize LEP against longitudinal (2nd Robinson) instabilities at higher beam current, an RF feedback system was introduced for fast amplitude and phase control of the RF voltage. The field probes of all eight cavities connected to one klystron are vector-added and used to control the klystron. Despite some initial technical difficulties, all klystrons are upgraded now with this system. A global voltage control system keeps the voltages at the four RF locations around the ring as equal as possible. It also reacts immediately when one klystron trips, trying to compensate the voltage loss by increase of 'close' modules or powering up idling ones.

One problem only showed up when the beam current was increased: the so-called electroacoustic (or ponderomotive) [11] oscillations. Electromagnetic forces change the cavity shape and therefore the resonance frequency of the cavity; furthermore, the beam induced voltage stimulates the tuning system to detune the cavity off resonance. Such a system can - and does in our case - start to auto-oscillate. Since all eight cavities oscillate independently, corrections through the single klystron cannot work. The only way to circumvent this problem is to keep the cavity, despite beam loading, on resonance. The price to pay is an increase in RF power consumption by a few percent. Recently a system using feedback and feedforward in the tuner control loop was tested successfully and is likely to be applied in LEP.

\section{ACHIEVEMENTS AND FUTURE}

The first $\mathrm{Nb} / \mathrm{Cu}$ industry module was received at CERN at the end of 1992 and from then on a steady flow arrived. Modules were reception tested, equipped with couplers, high power tested and stored; during shutdown periods, modules were installed in LEP. For the first time LEP was operated at $65 \mathrm{GeV}$ in 1995 to run in this completely new system. At start up 1996 the $\mathrm{W}^{ \pm}$-threshold energy was reached, allowing the production of $\mathrm{W}^{ \pm}$-pairs for the first time in LEP. The corresponding events in the detectors confirmed this achievement. After adding another 28 cavities in a short technical stop, LEP was run at $88.5 \mathrm{GeV}$ without any real problems and produced thousands of $\mathrm{W}^{ \pm}$-pairs. From then on during two winter shutdowns and a dedicated technical stop more modules have been installed increasing the top energy correspondingly, the system running always smoothly without any real problems. Only $8 \%$ of the total machine down time were directly due to the superconducting RF in 1997 ('98 announces similarly), a remarkable achievement for an essential machine component, especially when considering that it applies a very recent technology on a large scale.

Table I : LEP upgrade: cavities and modules

\begin{tabular}{|c|c|c|}
\hline date & sc cavities & modules \\
\hline May-90 & 0 & 0 \\
Oct-95 & 60 & 15 \\
Jun-96 & 144 & 36 \\
Oct-96 & 172 & 43 \\
Sep-97 & 240 & 60 \\
May-98 & 272 & 68 \\
May-99 & 288 & 72 \\
\hline
\end{tabular}

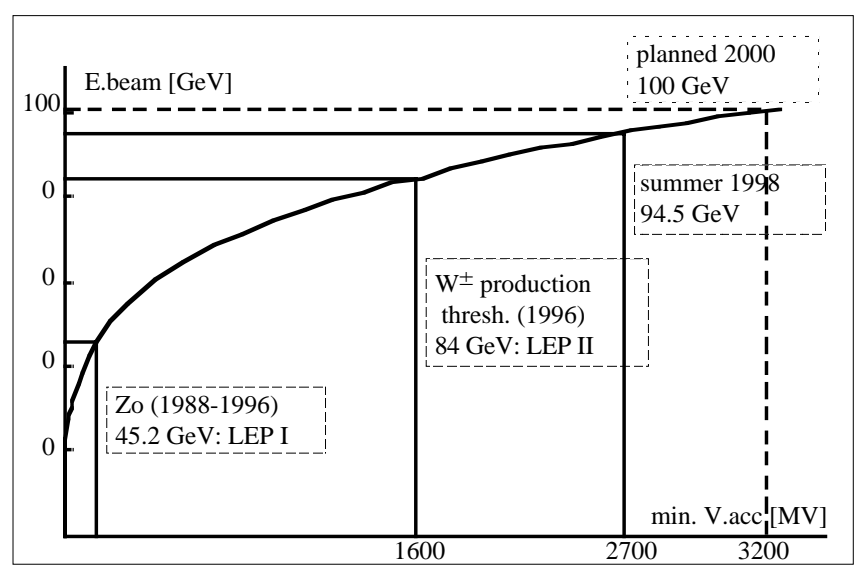

Fig. 8: LEP2 accelerating voltage and beam energy

At present about $2600 \mathrm{MV}$ accelerating voltage can be supplied by the superconducting cavities in LEP, by far the largest 'superconducting voltage' worldwide. Another $120 \mathrm{MV}$ are adjoined by the remaining 48 copper cavities from the first stage. A remarkable fact is that more than $10 \mathrm{MW}$ RF power is transmitted through the cryogenic environment of sc cavities to the beam with (nearly) no losses. Making use of all available voltage, a single beam has been stored at $96.5 \mathrm{GeV}$. Practically, a voltage reserve has to be kept to compensate rapidly for a tripping klystron, and thus $94.5 \mathrm{GeV}$ is the operational beam energy in 1998 with a total beam current of up to $6 \mathrm{~mA}$.

A further increase of the beam energy is in preparation, making use of the last bits of existing hardware (e.g. spare cavities) and pushing operational settings to the very limit for a final run in the year 2000 , dedicated to the search for new 
physics ground, especially the Higgs boson. If all the many necessary conditions - not only the voltage of about $3200 \mathrm{MV}$ - work out as hoped, $100 \mathrm{GeV}$ is in reach.

\section{A. Pending Problems}

An unexpected problem showed up on modules taken out of the ring to upgrade the HOM power lines. Some probe antenna cables had been overheated in the region where they were covered by superinsulation on both sides. Different indications point to directly picked up very high frequency components of the beam spectrum. To avoid burning further cables, the total LEP beam current is restricted today to $6 \mathrm{~mA}$. The bunch length is carefully monitored as well as the picked up power on the cables. During the next shutdown modules will be opened in the ring - exploiting the flexible cryostat design - to exchange the thin cables against thicker ones running outside the superinsulation layers

To achieve the highest possible energy in the year 2000, all cavities have to be pushed to their maximum. There are several cavities starting field emission above their design field and some of the older bulk $\mathrm{Nb}$ cavities show hard quenches. The fact that eight cavities are fed by the same klystron imposes a limit on all when a single one runs into an obstacle and therefore the relatively large scatter in field amplitude within such a group of eight such cavities is annoying. Extensive examinations have been done and several causes can be pointed at: 1) the coupling strength of the main couplers has a scatter due to the field flatness imprecision of the cavities and perhaps other mechanical tolerances, 2) the magic $\mathrm{T}$ power splitters are not exactly symmetrical causing cross-talk, 3) high power loads have reflections, 4) despite equal mechanical length of the wave guides, the electrical length from klystron to the cavities has a scatter, 5) the electrical length of the doorknob transition has a scatter.

1) and 2) cause already unequal fields without beam. $\lambda / 4$ transformers - realized as metal slabs in the wave guide, transformation ratio defined by their height - were installed in the wave guides of a few of the worst cases showing good results. The reflection at the RF loads will be reduced by improved matching. Different electrical lengths caused by 4) and 5) provoke different phases of the RF field, thus some cavities interact more than others with the beam; at injection where the synchronous phase angle is close to zero - there are even cavities sucking energy from the beam. In adding spacers the electrical length was made uniform in several modules with good success. To prepare the top energy run, the remaining (really) bad candidates will also be corrected in the coming shutdown periods.

\section{CONCLUSIONS}

The superconducting LEP RF system was initially planned for a gradient of $3 \mathrm{MV} / \mathrm{m}$, but specifications were increased to
5 and even $6 \mathrm{MV} / \mathrm{m}$ during the R\&D phase. Only some individual cavities do not reach this specification, but they are largely overcompensated by the much better performing ones. This gives rise to the hope that the system can be pushed even sensibly above specifications for the last year of LEP. The Q-values - inversely proportional to the $\mathrm{LHe}$ consumption - were asked to be at least $3.2 \times 10^{9}$. Also here only a few individual cavities do not perform precisely as specified, but are largely overcompensated by the others.

Ancillary equipment like tuners, power couplers, HOM couplers, insulation and cavity vacuum system and cryogenic supply work fully satisfactory. Initial technical problems like multipacting in the main couplers or electroacoustic oscillations have been overcome and now the whole system works so reliably that the down time due to the sc RF system is small compared to that due to more 'classical' faults. Routinely more than $10 \mathrm{MW}$ RF power are transmitted through this sc system to the beam. Evidently a well trained team is 'working in the background' permanently to repair small accessible defects and to do maintenance and upgrading work during the shutdown.

The resulting beam energy is largely sufficient to produce the desired $\mathrm{W}^{ \pm}$-pairs in large quantities - at present $94.5 \mathrm{GeV}$ are run routinely - and there is justified hope that $100 \mathrm{GeV}$ beam energy can be reached, thus closing the physics gap between LEP top and the future LHC lowest (equivalent) beam energy.

This achievement has only been possible due a large collaboration of many dedicated people from different groups: RF, cryogenics, vacuum, chemistry and surface treatment, mechanical workshops, general services, survey and civil engineering and not to forget outside companies. The remaining smaller problems are under examination and remedies are prepared. All the team hopes now that the last effort to push the beam energy to its ultimate limit will be rewarded by some new physics discovery.

\section{REFERENCES}

[1] LEP Design Report, CERN-LEP/84-01, June 1984

[2] LHC, CERN/AC/95-05 (LHC), Oct. 1995

[3] Ph. Bernard et al., Proc. 12th Int. Conf. on High-Energy Acc., Fermilab (1983) 244.

[4] E. Haebel, P. Marchand, J. Tückmantel, Proc. 2nd Workshop on RF SC, Geneva, Ed. H. Lengeler (1984) 281

[5] R. Stierlin, Proc. 3rd Workshop on RF SC, Argonne, Ed. K. Shepard, ANL-PHY-88-1 (1988), 639

[6] G. Cavallari et al. , id., p. 625

[7] E. Haebel , Proc. 5th Workshop on RF SC, DESY, Ed. D. Proch, 1991, p 334, DESY M-92-01

[8] E. Haebel et al., Proc. 6th Workshop on RF SC, IN2P3 (Saclay), Ed. B. Bonin, 1995, p. $707 \mathrm{ff}$

[9] J. Tückmantel et al., 'Improvements to Power Couplers for the LEP2 sc. Cavities ', Proc. IEEE PAC, Dallas, 1995

[10] E. Chiaveri, 'Large-scale Industrial Production of sc Cavities', Proc. 5th EPAC (Barcelona), 1996

[11] D. Boussard et al, Proc. 6th Workshop on RF SC, IN2P3 (Saclay), Ed. B. Bonin, 1995, p 641 